TREATMENT OF APRAXIA OF SPEECH IN ADULTS

\author{
John C. Rosenbek and Robert T. Wertz \\ Veterans Administration Hospitai, Madison
}

The purpose of this paper is to discuss therapeutic principles and specific procedures in the treatment of apraxia of speech. The sources of these principles and procedures are many and varied, and include previous clinical observations (Nathan, 1947; Goldstein, 1948; Johns, 1970), experimental research (Shankweiler and Harris, 1966; LaPointe, 1969; Johns and Darley, 1970; Dea 1, 1970), and the authors four years of work with a variety of apraxic patients.

\title{
General Principles
}

The very fact that an apraxic adult is brain damaged generates certain principles for therapy. Some of these principles are similar, if not identical, to those which have been emphasized for the aphasic. This is a fortunate economy so long as we continualiy remember that the forms for therapy and the specific methodology are quite different. The essential principles are:

1. All therapy activities should be organized according to task continua so that the patient does not struggle and so that he works at a high level of success during each session.

II. The brain damage in most instances will require that the patient take longer and work harder at regaining or relearning lost articulatory skills so the clinician should plan for intensive and extensive drill on stimulus-response pairs.

III. Because he is an adult with a history of normal speech and language comprehension-use, therapy should emphasize mastery of meaningfui and useful verbal communication early in therapy. With the severe patient, of course, periods of work on oral, nonverbal movements may be necessary.

IV. The brain damage may have imposed physiological limits on the patient's speech production mechanism. If so, 
the clinician should teach compensatory movements such as the intrusive schwa, equal and even stress, judicious use of the pause, and normal prolongation.

V. Efficient self-correction must be encouraged, although gentle reminders will be sufficient for some patients who appear to use it as a natural tendency.

VI. The apraxic adult will perseverate, so therapy should be designed which helps the patient to inhibit this behavior.

\section{Specific Principles}

Apraxia of speech is a nonlinguistic sensori-motor disorder of articulation characterized by impaired "capacity to program the positioning of speech musculature and the sequencing of muscle movements for the volitional production of phonemes" (Darley, 1969). This definition generates several specific therapeutic principles.

I. Therapy should concentrate on the disordered articulation and, therefore, be different from the 1 anguage stimulation and auditory and visual processing therapies appropriate to the aphasias.

II. Therapy should emphasize the relearning of adequate points of articulation and the sequencing of articulatory gestures.

III. Therapy should provide conditions such that the apraxic patient can advance from limited, automaticreactive speech to appropriate, volitional-purposive communication.

Apraxia of speech is characterized by inconsistency only insofar as error type is concerned. In general, a variety of phonetic conditions affect articulatory accuracy in predictable ways. These phonetic conditions are: manner of articulation, fricatives, affricatives, and consonant cTusters are more likely to be in error than vowels, nasals, and plosives; phoneme position, errors more likely in initial than final phoneme; difficulty of initial phoneme, word most likely to be in error if it begins with fricative, affricative, or consonant cluster; distance between successive phonemes, likelihood of error increases as dis tance between successive points of articulation within an utterance increases; word length, errors increase as words increase in length; word frequency, errors more likely on rare than on common words.

Therapeutic principles derived from these phonetic conditions are several: 
1. Stimulus items should be controlled for length, beginning with short reduplicating utterances, depending on the patient's severity, and systematically increasing stimulus length. Of course, work on several response lengths may go on simultaneously.

II. A hierarchial arrangement of phonemes and clusters as a basis for therapy is advisable, therapy to begin with easiest phonemes and advance systematically.

III. As phoneme sequencing becomes possible, stimuli should be selected so that the distance as determined by points of articulation between successive phonemes is small at first and progressively increased.

IV. If real words are used, these should be selected from a list of high frequency of occurrence words. However, response length, phoneme difficulty, and distance between successive phonemes are the most potent influences on articulatory accuracy and should be more carefully controlled.

V. Careful selection of stimuli with regard to initial phoneme will increase response adequacy and should, therefore, be considered in preparation of therapeutic materials.

VI. Teaching points of articulation for some phonemes in the final position of a VC syllable is advisable in
some cases.

Articulatory accuracy in apraxia of speech is influenced by mode of stimulus. Auditory-visual stimulation is better than auditory or visual alone--visual in this instance refers to watching the clinician as he speaks. Useful as well are written stimuli for many apraxic patients, especially those who are relatively free of a coexisting aphasia. Finaliy, apraxic patients, in general, achieve greater phonemic accuracy (at least at the single sound level) if they are allowed to monitor their own speech in a mirror. Principles of therapy, then, are obvious.

I. The integral stimulation method ("listen to me and watch me") is parsimonious, effective, and should be tried first.

II. As therapy progresses and the patient becomes able to produce volitional, nonimitative speech, first auditory then visual cues should be gradually faded.

III. Only after the integral stimulation method has failed with a patient or with a particular phoneme should the clinician move to other methods. Useful alternatives are phonetic placement, phonetic derivation,
and the "key word" method. 
IV. Developing a strong visual memory of correct production should be emphasized from "day one"; the mirror and written word are useful to this process.

\section{Case Report}

What follows is an outline for therapy which is only one of several possible prototypes, but the one with which we are most satisfied. The patient for whom this therapy was devised is a 58 year-old female with a six-year duration of apraxia of speech. A short period of therapy had ended in no progress five and one-half years before she first came to us. Her useful speech consisted of five or six automatic phrases and counting to five, omitting three. Aphasia was minimal, and there was no dysarthria.

Our goal was to help this lady program a few simple commands and social utterances, such as "I'm fine." We realized, of course, that the utterances we could expect would be tempered by her own particular repertoire of phonemes which, if one ignored phonemes present in her automatic speech, consisted of one, /a/, which she could produce imitatively. All other sounds were not stimulable during the first session. After five sessions, we had determined that she could, with very strong stimulation and several trials, also imitate $/ i /$, $/ a I /$, and /t/. We decided that one thrust of the therapy then would be to teach her "I want to eat." We emphasize the "one thrust" because we saw her eight times a week and worked on other sounds and utterances simultaneously. Let us examine just this one phrase, however, because it satisfies most of the criteria outlined previously, and it allows us to present our specific therapy techniques with adequate rationale.

The phrase "I want to eat" is useful, the words are short and of high frequency, the phonemes are visible, and any combination requires minimal excursions for proper points of articulation. Some of the phonemes were stimulable in isolation by the integral stimulation approach, and the phrase "want to" existed in a slightly different form, counting /wan tu/, in her preserved automatic speech.

While we broke the command down into several parts (/aI/, /wan $t u /, / i /, / t /)$ in the initial sessions, our method for these individual portions and for the utterance as a whole, was similar and followed the following steps.

Step I. Integral stimulation [look at me (a); watch me $(v)$,$] and simultaneous production (ciient-$ clinician saying it together).

Step II. Integral stimulation ( $\left.a, v_{1}\right)$ and delayed production (client saying it after clinician) with visual $\left(v_{7}\right)$ cue. 
Step III. Integral stimulation $\left(a, v_{1}\right)$ and delayed production with no $v_{1}$ cue.

Step IV. Integral stimulation and successive productions without intervening stimulation of auditory or visual $\left(v_{1}\right)$ cues.

Step V. Simultaneous production with written stimuli $\left(v_{2}\right)$ only. Patient reads the response from board or ${ }^{2}$ ards.

Step VI. Delayed production with $v_{2}$ cue only. In this condition, the stimulus is written and then erased or concealed before client attempts it.

Step VII. Appropriate response prompted by a question (in this case, "What do you say when it's $5: 30$ and you are hungry?").

Step VIII. Appropriate response in role playing situation when clinician, appearing as a nurse, walks into the therapy room.

Step IX. Appropriate response in true life situation.

\section{Discussion}

With each patient, we may not pass systematically through all steps for a variety of reasons. Obviously, at the single sound and, to a lesser extent, at the single word level, steps VII, VIII, and IX would be impossible. In other instances, the patient may be able to jump from Step IV to step IX. This is often the case with very simple utterances or with the moderately or mildly impaired patient. It is also the case when response generalization occurs as it sometimes does even with the severe patient, and he begins to engage in effective, efficient, searching, and self-corrective behavior. All these conditions which permit skipping of some steps should prompt the clinician to probe systematically for the target response while progressing through the steps.

Auditory discrimination does not precede production in the model out 1 ined--and for a very good reason. The apraxia of speech patient who is either pure or only mildiy aphasic does not need discrimination training as Aten, Johns, and Darley (1970) have demonstrated; nor does the model emphasize a motokinesthetic approach, because deficits in oral sensation and perception have been demonstrated in patients with apraxia of speech (Rosenbek, 1970; Larimore, 1970). Trial therapy employing this approach can be utilized if integral stimulation has failed and if oral sensation and perception is intact at the time of diagnosis. It does emphasize the auditory and visual modalities, especially the visual, because these appear to be most potent in guiding the articulators. While there 
is no experimental evidence, establishing or strengthening "visual memory" appears extremely important to therapeutic

Because the outline would become burdensome, several slight modifications of our nine-step procedure were omitted; however, they should be mentioned. At the utterance level, all work at this level until same control. Rather than delaying managed with equal rel individual words or sounds can be Theility, the clinician should make others and accepting these include cueing some sounds and not if these do not adversely For example, omissions in affect the overall communication. noticed in the speech of normals and position often go unspeech of the apraxic if failure and may be accepted in the delay the patient's using a few meaning permit these errors would Telegraphic utterances also should aningful, useful utterances. omitted words are in the patient's be accepted unless the

Other modifications not reflected in the nine steps include variations in the clinician's stimulus presentation. In general, stimuli are presented slowly with pauses between some words, with prolongation of some consonants and vowels, and with use of an intrusive schwa to break up consonant ciusters. Some parts of an utterance may be produced louder and/or with exaggerated articulatory gestures. Some may be presented musically or with a particular rhythm pattern. For example, the contraction / aIm/ was practiced for weeks as al: m:, with a rising and falling inflection. In other instances, instead of putting a pause between words, they may be combined as a single utterance--a condition which is closer to normal production. For example, /wan tu/ which became /wantu/ was always rehearsed as a single word. The rule is, "Do what increases the patient's response adequacy."

Some sounds may not respond to an integral stimulation method--at least initially. The clinician, therefore, should be versatile. Phonetic placement is a useful method, especially for the front sounds. The phoneme /f/ entered our patient's repertoire after four days of placing her lower 1 ip between her teeth, guiding her mouth closed in a bite, and then asking her to "biow." At one point, gesturing toward her mouth was sufficient to get her to produce an acceptable /f/.

Singing seems to facilitate speech in some apraxic patients. In our patient, it was the only way we could get her to produce an /1/: / Iz//12/ing to "Jingle Bells" was most effective. For the milder patient with a large phoneme repertoire, singing may facilitate whole phrases and sentences.

A variation on the "key word" method is also useful. Previously mentioned was the rather easy modification of /wan tu/ to/wantu/. It is sometimes possible to dissect a 
sound from a "key word," usually an automatic response, and then combine it with other phonemes. The sound /g/ existed only in /gud/. By having out patient say /gud//gud/, /gou/, we have arrived at a point where she now produces the $/ g /$ about $20 \%$ of the time without a preceding/gud/.

By no means have we presented all the possibilities in apraxia therapeutics. This therapy admits to wide individual differences prompted both by client and clinician. Instead, we have attempted to provide a skeletal framework for therapy in the hope that individual clinicians will provide the modifications and nuances, the flesh and blood of any successful therapy. Finaliy, we offer these principles and methods not so much as answers to the sometimes perplexing questions of apraxia therapeutics but as developmental milestones so that we can look back one day and see how far we
have come.

\section{REFERENCES}

Aten, J. L., Johns, D. F., and Darley, F. L. Auditory perception of sequenced words in apraxia of speech. Speech Hearing Res., 14, 1971, 131-143.

Darley, F. L. Notes prepared for delivery to American Speech and Hearing Association Convention, Chicago, 1969.

Deal, J. L. The influence of linguistic and situational variables on phonemic accuracy in apraxia of speech. Unpublished Ph.D. Dissertation, University of Southern Mississippi, 1970 .

Goldstein, K. Language and Language Disturbances. New York: Grune and Stratton, $\frac{19}{19} \overline{8}$.

Johns, D. F. Treatment of apraxia of speech. Paper presented to the American Speech and Hearing Association National Convention, New York, 1970.

Johns, D. F. and Darley, F. L. Phonemic variability in apraxia of speech. J. Speech Hearing Res., 13, 1970,556-583.

La Pointe, L.L. An investigation of isolated oral movements, oral motor sequencing abilities, and articulation of brain-injred adults. Unpublished Ph.D. Dissertation, University of Colorado, 1969.

Larimore, H. W. Some verbal and non-verbal factors associated with apraxia of speech. Ph.D. Dissertation, University of Denver, 1970.

Nathan, P. W. Facial apraxia and apraxic dysarthria. Brain,
70, ig47, 449-478. 


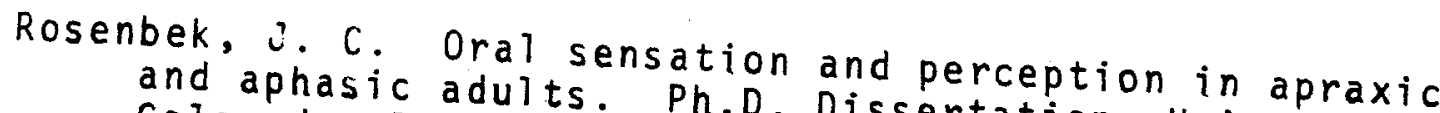
Colorado, 1970 .

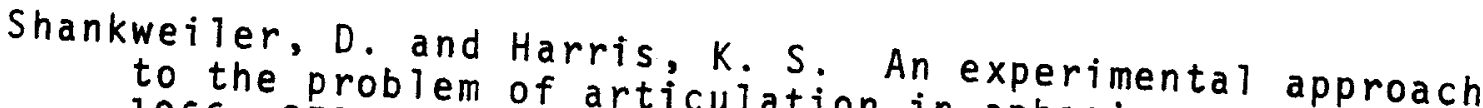
$1966,272-292$. 